\title{
A NOVEL FABRICATION ME'THOD FOR SUSPENDED HIGH-ASPECT-RATIO MEMS STRUCTURES
}

\author{
Wen-Cheng Kuo and Yao-Joe Yang \\ Department of Mechanical Engineering \\ National Taiwan University, Taiwan, ROC \\ e-mail: yjy@ntu.edu.tw
}

\begin{abstract}
The high-aspect-ratio structures (HARS) are widely used for MEMS devices such as micro-gyroscope, microaccelerometer, optical fiber switches, and so on. Various fabrication methods, such as SOI [1] SCREAM [2] and SBM [3] processes, were proposed to fabricate HARS. However, these methods are focus on the fabricating suspended microstructures with small trench openings (e.g., less than $10 \mathrm{fm}$ ), which often limits the maximum inplane displacement of the structures. In this paper, we propose a low-cost single-mask, single ICP-RIE process for fabricating suspended MEMS structures which have the characteristics of arbitrary sizes of trench openings, very high trench aspect ratio (about 20) and very large structure thickness (about $100 \mathrm{\mu m}$ ). Also, the proposed process potentially can be used to fabricate the devices for large inplane-displacement applications.
\end{abstract}

Keywords: Inductively coupled plasma (ICP), high aspect ratio structure (HARS), deep reactive ion etching (DRIE)

\section{INTRODUCTION}

It is well known that single-crystal silicon (SCS) is an excellent material for MEMS devices because of its superior mechanical and thermal characteristics. Therefore, many MEMS devices, such as micro-gyroscopes, accelerometcrs, and optical mirrors/shutters/switches, take advantages of the well-established properties of SCS with a relatively large device thickness to improve device performance. The devices of this type are also classified as suspended high-aspect-ratio structures (suspended HARS).

For creating suspended HARS, there are many approaches, such as SOI [1], SCREAM [2], SBM [3] processes. The SOI process uses the buried oxide layer as the sacrificial layer for wet releasing. This process is quite straightforward for structure releasing and is easy to control. However, the major disadvantage of this process is that the wafer cost is about two orders higher than that of standard silicon wafers.
As for the other processes, extra deposition or ionimplantation steps are needed for creating the layers (e.g., silicon oxide, polymer, or heavy pin-type doping) that will be used to protect the sidewalls of suspended structures during the releasing step. Therefore, for these processes, it is quite difficult to successfully fabricate suspended HARS if the maximum width of trench opening is relatively large. The explanation is described as follows: Fig. 1 shows the typical process steps for fabricating suspended HARS using sidewall protection layers. Step 1 is the patteming for ICP etching mask Step 2 is the trench etching. Before releasing the suspended structure by isotropic silicon etching, the sidewalls of the structure have to be protected by depositing passivation layers (polymer or oxide), as shown in Step 3. Since $W_{1}$ is much greater than $W_{2}$, the trench aspect ratio in Region $I$ is much less than that in Region III. Note that in this work we define the trench aspect ratio as the ratio of trench depth to trench opening width. Therefore, the deposition rate in Region I is higher than that in Region III due to the conductance effect [4] and the reactant transport effect [5]. As a result, the passivation layer in Region I is thicker than that in Region III. On the other hand, the thickness of the passivation layer in Region II is very close to that in Region I. In Step 4, the passivation layer in the bottom of each trench is removed so that the suspended structure can be ready to be released by isotropic etching. This step will also remove part of the passivation layer on trench tops. However, as shown in the figure, since the thickness of the passivation layers in Regions I and II are quite close, it is very frequent that the etching of Step 4 also creates the unwanted openings on the top edges of trenches, especially when the passivation layers in Region I have to be removed completely. These umwanted openings result in the damages of the suspended structures during the structure-releasing process, as shown in Step 5.

The typical process to avoid the unwanted openings is to minimize the widths of the trench openings so that the thickness of the passivation layer on the bottom floor of each trench will be smaller than that in Region II. It is easier to remove the passivation layer on the bottom, but only part of passivation layer in Region II is removed. Therefore, the maximum in-plane displacement of the suspended structures will be limited with small trench width. 

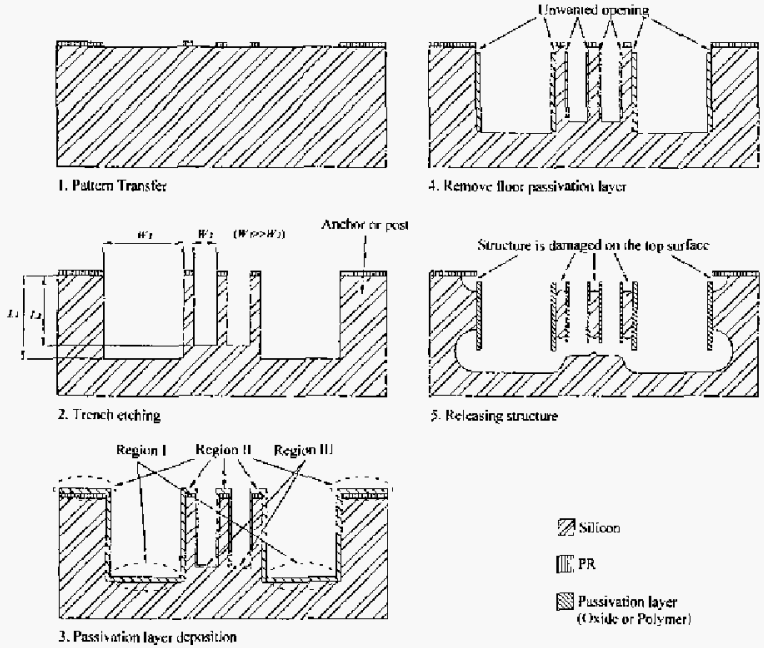

5. Rekeasing structure

3.Passintion

Asilicon

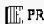

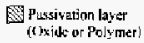

Fig. 1. The failure mechanism caused by unwanted opening.

In this work, we present an ICP-RIE process that employs the characteristic of the microtrenching effect [6] to fabricate suspended HARS. The microtrenching effect can be enhanced by using special ICP etching parameters. Since the proposed fabrication process has no constraints on the design of the width of trench openings, the fabricated devices by this process can be used for the applications which require large in-plane displacement. Furthermore, all the process steps can be integrated as a single-run single-mask ICP-RIE process, which makes this proposed technique simple and inexpensive.

\section{FABRICATION}

Fig. 2 shows the 5 -step fabrication process proposed in this work. Step 1 is in fact the standard ASE [7] process. The exposed silicon is then etched to a desired depth. Step 2 is similar to the passivation step of the standard ASE process, except that the time for depositing the passivation layer is about few minutes. The purpose of this step is to gencrate the polymer around the structures. Because of the conductance effect [4] and the reactant transport effect [5], the polymer deposition rate is decided by the width of trench opening and the trench aspect ratio. The larger the width of trench opening, the larger the polymer deposition rate at the trench bottom. Therefore, the polymer thickness in Region IV is thicker than Region VI. Note that the polymer thickness in Region $\mathrm{V}$ is similar to that in Region IV. Step 3 is similar to the etching step of the standard ASE process, except that the time for floor polymer removing is about few minutes. The floor polymer layers in Region IX can be easily removed completely because they are thinner. On the other hand, because of the microtrenching effect [6], the etching rate at the corners of a trench bottom is larger than that on the center of the trench bottom. Therefore, for the regions with smaller trench aspect ratio or with larger width of trench openings (i.e., floor polymer layers are relatively thicker in these regions), only the polymer on the corners of a trench bottom is removed (as indicated by Region VII). The polymer etching time has to be controlled carefully so that the polymer in Region IX is removed completely and only part of the polymer in Region VIII is removed, which in turn avoids the umwanted openings on the top edges of each structure. In Step 4, the exposed silicon is isotropically etched by using the ASE etching step without any platen power. The purpose of this step is to release structures. In Step 5, oxygen plasma is used to strip photoresist and polymer.
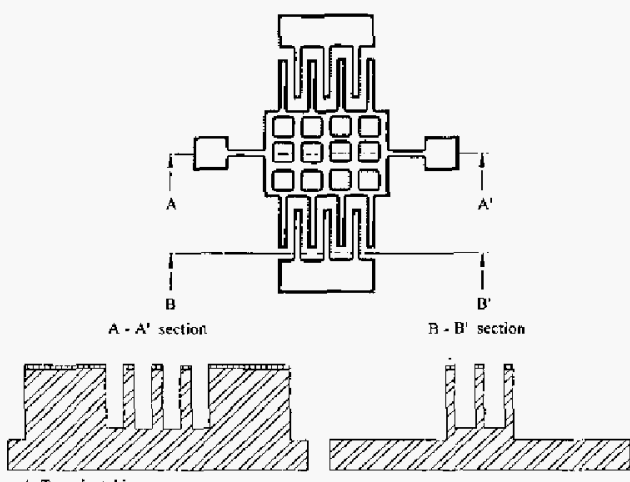

1. Tueneh etching
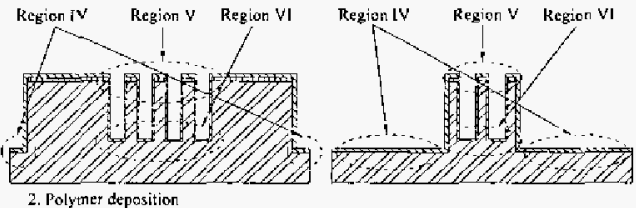

Rețion VII Region VIII Region $1 \mathrm{X}$
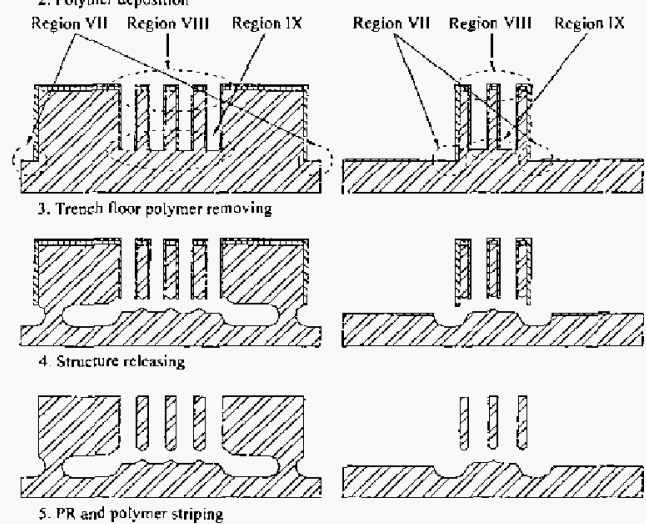

6

5. PR and polvmer striping

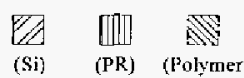

Fig. 2. Fabrication process.

TRANSDUCERS'05

The 13th International Conference on Solid-State Sensors, Actuators and Microsystems, Seoul, Korea, June 5-9, 2005 


\section{PROCESS CONSIDERATIONS AND RESULTS}

\section{A. Considerations for Polymer Deposition Time:}

The process uses a polymer layer to avoid sidewall erosion during the following floor polymer removing step and structure releasing step. Due to the conductance effect [4] and the reactant transport effect [5], the flux distribution of CF-based species around a trench profile is affected by the width of trench opening and the aspect ratio of trench. For the cases that the width of trench opening is large or that the trench aspect ratio is small, the polymer deposition rate is large at trench bottom. As shown in Step 2 of Fig. 2, because the trench aspect ratio on Region IV is smaller than that in Region VI, the bottom passivation layer thickness in Region IV is thicker than that in Region $\mathrm{VI}$, and is almost the same as the thickness at Region V.

If the polymer deposition time is relatively short (less than 15 minutes), the differences of polymer thickness between Region IV, V, and VI are small. When removing the floor polymer at Region VI, the time must be very accurate. Otherwise, it is very likely that the polymer layer at Region V will also be removed, and then the "unwanted openings" are created. On the other hand, if the deposition time is relatively large (greater than 20 minutes), the difference of polymer thickness in Regions VI and V will be relatively large, which implies that the difference of the times for removing the polymer at Region $\mathrm{V} 1$ and $\mathrm{V}$ is also large. Under this condition, it is much easier to control the etching time for achieving the desired etching condition in which the floor polymer is effectively removed without creating the "unwanted openings".

\section{B. Considerations for Floor Polymer Removing}

This step (Step 3 in Fig.2) is a combination of physical (ion) bombardment and chemical reaction using fluorine radicals. Only the ion bombardment is dominant for this etching step [8]. Therefore, the probability of ion bombardment on sidewall polymer has to be reduced so that the sidewall polymer will not be etched simultaneously. However, because of the microtrenching effect [6], the etching rate at the corners of a trench bottom is larger than that on the center of the trench bottom. It is believed that the microtrenching effect is produced by the impact of high-energy particles on the sidewalls followed by specular reflection, which in turn leads to a "focusing" of high energy particles at the base of the sidewalls, giving rise to higher etch rates. Our experiment shows that the microtrenching effect can be enhanced by increasing the platen power and reducing the APC angle (i.e. reducing pressure).

\section{Considerations for Structure Thickness:}

For the same pattern design (i.e., using the same mask), increasing structure thickness also increases the trench aspect ratio. Therefore, the process parameters for the steps of polymer deposition and structure releasing must also be modified. In the polymer deposition step, the CF deposition rate near the trench bottom decreases as the trench aspect ratio increases. In order to retain sufficient thickness of polymer on the sidewalls of trenches, the polymer deposition time should also be increased with the trench aspect ratio. Besides, due to the conductance effect [4] and the reactant transport effect [5], the flux distribution of etchants around a trench profile is affected by the width of trench opening and the aspect ratio of trench. Hence, in the structure-releasing step, the lateral etching rate decreases as the trench aspect ratio increases. Therefore, the etching time for structure releasing also increases with the trench aspect ratio.

\section{Process Results:}

Fig. 3 shows the SEM picture for a micro-relay. The structure thickness is $50 \mu \mathrm{m}$, and the maximum trench aspect ratio is 10. Fig. 4 shows the comb-drive actuator fabricated by the proposed process. The structure is 100 $\mu m$ in thickness, and the maximum trench aspect ratio (trench depth over trench opening) is 20 . Fig.5 shows a suspended comb-drive actuator which can be used for large-displacement motions (over $100 \mu \mathrm{m}$ ). The thickness of the actuator is $100 \mathrm{\mu m}$, the beam width is $15 \mathrm{\mu m}$ and the length of the maximum suspended beam is $2 \mathrm{~mm}$.

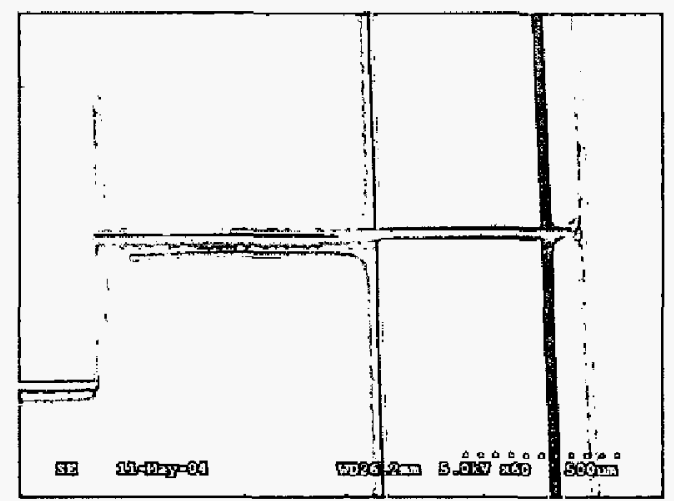

Fig. 3. Fabricated micro-relay with $50 \mathrm{\mu m}$ in thickness. 


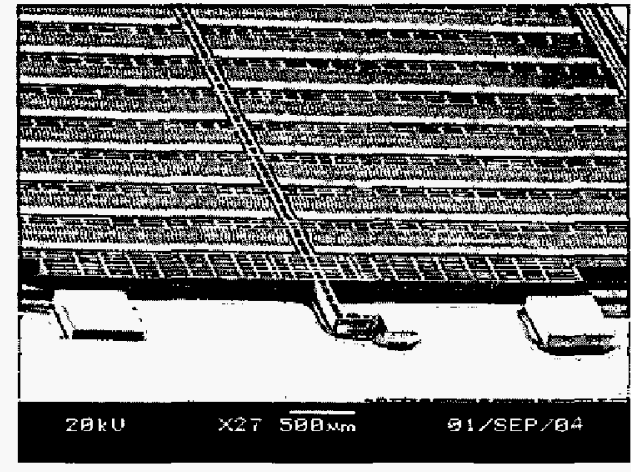

(a)

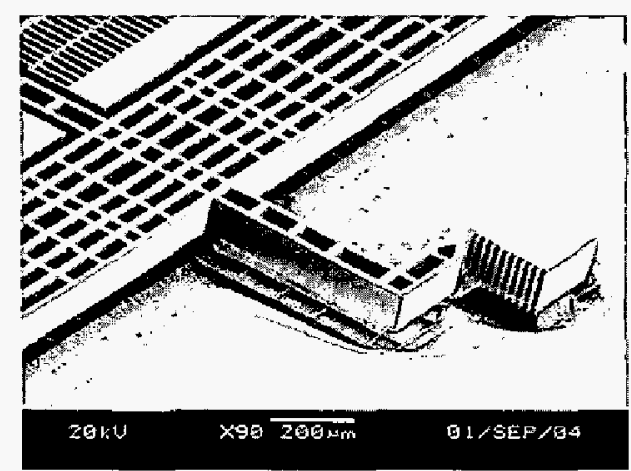

(b)

Fig. 4. (a) the comb-drive structure with $100 \mu \mathrm{m}$ in thickness (b) the close-up view of the structure.

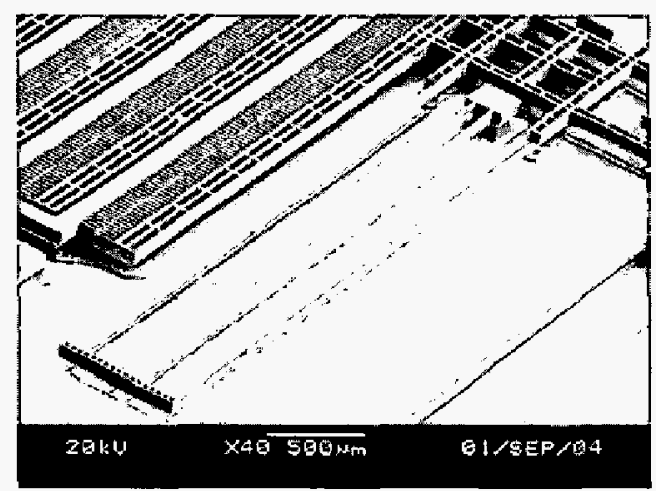

Fig. 5. The long suspended folded beam with $2 \mathrm{~mm}$ in length, $15 \mu \mathrm{m}$ in width.

\section{CONCLUSION}

In this work, we present a novel process for fabricating high-aspect-ratio suspended structures with large width of trench openings. By enhancing the microtrenching effect, we successfully released suspended structures without thoroughly removing the floor polymer at regions with small trench aspect ratio. The steps for trench etching, sidewall passivation, structure releasing, and $\mathrm{PR} /$ polymer striping can be integrated as a single-run single-mask $1 \mathrm{CP}$ RIE process, which effectively reduces the process complexity and fabrication cost. By using the proposed process, suspended structures with the maximum thickness of $100 \mu \mathrm{m}$ and the maximum aspect ratio of 20 are demonstrated. Also, the proposed process can be used to fabricate the devices for large in-plane-displacement applications.

\section{Acknowledgements}

This work is supported by the National Science Council, Taiwan, R.O.C. (Contract No: NSC 932212 E 002 023). The authors would like to thank Mr. Hsin-Hong Liao for his help on fabrication process.

\section{References}

1. P. F. Indermuehle, C. Linder, J. Brugger, V. P. Jaecklin, and N. F. de Rooij, "Design and fabrication of an overhanging $X Y$-microactuator with integrated tip for scanning surface profiling", Sensors and Actuators A, Vol. 43, 1994, pp. 346-350.

2. K. A. Shaw, Z. L. Zhang, and N. C. MacDonald, "SCREAM I; a Single mask, single-crystal silicon, reactive ion etching process for microelectromechanical structures", Sensors and Actuators A, Vol. 40, 1994, pp. 63-70.

3. S. Lee, S. Park, and D. Cho, "The Surface/Bulk Micromachining (SBM) process: a new method for fabrication released MEMS in single crystal silicon", Journal of microelectromechanical Systems, Vol. 8, No. 4, 1999, pp. 409-416.

4. S. Dushman and J. M. Lafferty, Scientific Foundations of Vacuum Technique, $2^{\text {nd }}$ ed., Wiley, New York, 1962, p94.

5. J. C. Arnold, D. C. Gary, and H. H. Sawin, "Influence of reactant transport on fluorine reactive ion etching", Journal of Vacuum. Science \& Technology B, Vol. 11, 1993, pp. 207 I-2080.

6. R. J. Hoekstra and M. J. Kushner, "Microtrenching resulting from specular reflection during chlorine etching of silicon", Journal of Vacuum Science \& Technology B, Vol. 16, No. 4, 1998, pp. 2102-2104.

7. F. Laermer and A. Schilp, "Method of anisotropically etching silicon", USA Patent No. 5501893.

8. I. W. Rangelow, "Dry etching-based silicon micromachining for MEMS", Vacuum, Vol, 62, 2001, pp. 279-291. 